\title{
O ENSINO DE SISTEMAS DE CONTROLE POR MEIO DA UTILIZAÇÃO DE UMA PLANTA TÉRMICA E TRANSFORMADAS DE LAPLACE
}

\author{
THE TEACHING OF CONTROLE SYSTEMS THROUGH THE USE OS THERMAL PLANT AND
}

LAPLACE TRANSFORMS

Mateus Vorpagel ${ }^{1}$, Italo Gabriel Neide ${ }^{2}$, Juliano Schirmbeck ${ }^{3}$

DOI: 10.37702/REE2236-0158.v40p449-461.2021

\section{RESUMO}

Neste trabalho foi desenvolvida e analisada uma sequência didática baseada na metodologia POE, também conhecida como metodologia da Previsão, Observação e Explicação, para o ensino de sistemas de controle de primeira ordem da disciplina de Sinais e Sistemas do curso de Engenharia Elétrica. Esta pesquisa foi realizada em uma universidade comunitária no município de Lajeado, Rio Grande do Sul, e teve a participação de vinte e sete acadêmicos do quinto semestre do curso de Engenharia Elétrica. De modo a alcançar os objetivos propostos, utilizou-se as atividades experimentais como estratégia para a aprendizagem desse conteúdo. A principal atividade que foi utilizada nesta pesquisa foi a planta térmica, na qual foi simulado um sistema de controle de primeira ordem, aplicando-se, posteriormente, as Transformadas de Laplace. Os dados analisados indicaram que os acadêmicos possuíam bons conhecimentos prévios sobre sinais e sistemas, todavia faltavalhes a integração de conhecimento entre a teoria de controle e os cálculos matemáticos. A elaboração das atividades experimentais, focadas tanto na teoria quanto na prática, possibilitou que os estudantes conseguissem desenvolver habilidades e competências capazes de realizar de forma autônoma problemas de controle de sistemas. A metodologia POE se fez presente em todas as etapas, o que favoreceu a aprendizagem e consolidou a parceria entre alunos e professor na busca do conhecimento.

Palavras-chave: metodologia POE; Sinais e Sistemas; atividades experimentais.

\begin{abstract}
In this work, a didactic sequence based on the POE methodology was developed and analyzed, also known as the Forecast, Observation and Explanation methodology, for the teaching of first order control systems in the subject of Signals and Systems in the Electrical Engineering course. This research was carried out at a private university in the city of Lajeado, Rio Grande do Sul and had the participation of twenty-seven academics from the fifth semester of the Electrical Engineering course. In order to achieve the proposed objectives, experimental activities were used as a strategy for learning this content. The main activity that was used in this research was the thermal plant, in which a first-order control system was simulated, subsequently applying the Laplace Transforms. The analyzed data indicated that the students had good previous knowledge about signals and systems, however, they lacked the integration of knowledge between control theory and mathematical calculations. The development of experimental activities, focused on both theory and practice, enabled students to develop skills and competences capable of autonomously performing system

\footnotetext{
1 Universidade do Vale do Taquari - Univates, Programa de Pós-graduação em Ensino de Ciências Exatas PPGECE, vorpa2008@gmail.com

2 Universidade do Vale do Taquari - Univates, Programa de Pós-graduação em Ensino de Ciências Exatas PPGECE, italo.neide@univates.br

3 Universidade do Vale do Taquari - Univates, Programa de Pós-graduação em Ensino de Ciências Exatas PPGECE, juliano.schirmbeck@univates.br
} 
control problems. The POE methodology was present at all stages, which favored learning and consolidated the partnership between students and professors in the pursuit of knowledge.

Keywords: POE Methodology; Signals and Systems; experimental activities.

\section{INTRODUÇÃO}

As engenharias, que são cursos de ensino superior voltados para a área da tecnologia, têm como objetivo integrar a teoria à prática. Demo (1998) afirma que não há como realizar a prática sem ter como base a teoria, da mesma forma que não se pode obter a teoria sem a prática em sala de aula. Entretanto, laboratórios modernos e inovadores, dependendo da área escolhida para seguir, têm custos elevados. Assim, conforme Demo (1998) nos inspira, professores criativos conseguem produzir ótimos conteúdos e materiais didáticos para suas aulas, utilizando poucos recursos financeiros. Dessa forma, conseguem motivar seus alunos durante o processo de ensino e aprendizagem.

Teodoro e Veit (2002) afirmam que a utilização de tecnologias como um agente facilitador nos processos de ensino e de aprendizagem tem sido alvo de grande interesse pelos pesquisadores das teorias de ensino. Entretanto, não basta apenas trazer as tecnologias de ensino para a sala de aula sem o uso de uma metodologia adequada. Desse modo, este trabalho foi desenvolvido com base na metodologia POE, conhecida como metodologia da Previsão, Observação e Explicação. Alexander (2010) afirma que a POE inicialmente foi criada para ser um método de ensino que avaliasse a aprendizagem dos alunos ao longo de um curso de ciências exatas, não atribuindo notas. Porém, ela é muito mais do que isso, sendo considerada uma metodologia ativa.

Sasaki e De Jesus (2017) recomendam que essa metodologia seja trabalhada em pequenos grupos de alunos, pois favorece a interação e o compartilhamento de ideias entre os pares. De acordo com os autores, ela demanda a demonstração de um experimento de característica qualitativa, sendo dividida em três etapas distintas. Na primeira, o aluno fará suas previsões sobre um determinado evento, justificando-o com seus conhecimentos prévios. $\mathrm{Na}$ segunda, o estudante deverá realizar o experimento proposto, comparando-o com suas previsões. Já na etapa da explicação, o discente procura explicar as diferenças e semelhanças encontradas nas etapas anteriores, fazendo com que haja discussões, criando discrepâncias entre as ideias iniciais e o resultado observado durante a aplicação da metodologia.

Nos cursos de engenharia, desafiar os estudantes constantemente, promovendo situações reais e práticas dentro da sala de aula, é importante para o desenvolvimento acadêmico e profissional. A área de controle é marcada, principalmente, pela matemática abstrata e complexa, sendo que, com a utilização da metodologia de aprendizagem POE, buscou-se a integração entre as tecnologias e as atividades experimentais.

O principal objetivo desta pesquisa foi desenvolver e analisar uma sequência didática por meio da utilização da metodologia POE para o ensino de sistemas de controle de primeira ordem na disciplina de Sinais e Sistemas. Durante a pesquisa, os conhecimentos prévios dos estudantes relacionados a esse conteúdo foram verificados, bem como a aplicação do conteúdo em situações do seu cotidiano. Utilizou-se atividades experimentais e computacionais para o desenvolvimento da metodologia POE, a partir da análise do desenvolvimento da intervenção por meio de metodologia descritiva, identificando-se os possíveis impactos neste trabalho. O trabalho também permitiu conhecer as percepções dos alunos frente às atividades desenvolvidas. 


\section{REFERENCIAL TEÓRICO}

Nesta seção serão apresentadas características relevantes para este trabalho das tecnologias de ensino, a teoria de sistemas de controle de processos e a fundamentação da Metodologia POE (Previsão, Observação e Explicação).

\section{Tecnologias de Ensino}

De acordo com Dos Santos, Neide e Rehfeldt (2017), as tecnologias de ensino facilitam o processo de ensino e aprendizagem.

O emprego de softwares interativos possibilita a coordenação de múltiplas representações, pois permite várias visualizações. Na área de controle existe o $\mathrm{Matlab}^{4}$, que reproduz complexos sistemas de controle e modelagem.

As tecnologias servem de base para que os docentes desenvolvam a prática de ensinar modelando. Há objetos de aprendizagem para dar suporte aos professores em sala de aula, a saber: os jogos digitais, os simuladores e a modelagem computacional (NEIDE; QUARTIERI, 2016).

Como exemplos de softwares de modelagem computacional mencionamos: o GeoGebra $^{5}$, que possibilita a construção de gráficos e funções matemáticas, representandoas no plano cartesiano; e o Matlab, que resolve complexos problemas físicos e matemáticos com base em sua linguagem de programação própria.

Outra ferramenta utilizada frequentemente é a plataforma Arduino. Segundo Banzi (2011), Arduino é uma plataforma de computação física de programação aberta, formada por um microcontrolador com entradas e saídas digitais, empregada em diversos segmentos, desde a captura de dados para o computador até o controle de processos industriais.

\section{Teorias de Sistemas de Controle de Processos}

Segundo Ogata e Yang (2002), o controle automático de processos é essencial para as áreas da engenharia e da ciência, por exemplo: o controle de velocidade de cruzeiro de veículos automotores; sistemas robóticos e de manufatura; e o controle industrial de temperatura e umidade.

Conforme Ogata e Yang (2002), o processo é toda operação a ser controlada, dentro do sistema. O sistema é a integração de diversos componentes que trabalham em conjunto para alcançar um objetivo comum. Já o distúrbio, que pode ser interno ou externo, é um sinal que pode modificar o valor da variável controlada do sistema. No final se determina o controle com realimentação, como uma operação que, com distúrbios não previsíveis, reduz a diferença entre o sinal de entrada e saída do sistema, quando opera nessa diferença.

Foi apresentado um sistema de controle de velocidade, regulador Watt de velocidade, para um motor a combustão, que possui um regulador de velocidade que controla a quantidade de combustível inserida dentro da câmara de combustão do motor por meio de uma válvula de controle. Caso a velocidade caia em função de algum distúrbio, o regulador de velocidade remete um sinal para a válvula abrir e aumentar a velocidade do motor até a velocidade desejada.

O sistema de controle de temperatura traduz-se em uma interface para regular a temperatura de um sistema com base num controlador de temperatura, que recebe um sinal de entrada por meio de um sensor analógico ou digital, que o compara com o valor configurado. O controlador remete um sinal de saída para acionar um componente que permite atingir a temperatura desejada, que pode ser tanto de resfriamento ou aquecimento.

O sistema de controle empresarial constitui-se nos grupos de gerência, pesquisa, desenvolvimento, projeto preliminar, experimentos, projeto e desenho de produto,

\footnotetext{
4 Mais informações a respeito do software em https://www.mathworks.com/products/matlab.html.
}

5 Mais informações a respeito do software em
https://www.geogebra.org/?lang=pt. 
fabricação, montagem e testes. As características de cada grupo devem ser aplicadas por meio de equações. Como o sistema é de malha fechada, os distúrbios são considerados como carência de matéria-prima ou de mão de obra, interrupção de comunicação e erros humanos. Já o desempenho pode ser verificado por meio da relação entre a realização progressiva e o tempo.

Os sistemas de controle são segmentados em controle de malha aberta e fechada. No sistema de malha aberta a saída não é comparada com a entrada, sendo que cada sinal de entrada é responsável por uma única condição de operação, que deve estar calibrada corretamente para não comprometer o resultado da operação; por exemplo, o controle de tráfego por meio de sinaleiras. Já o sistema de controle de malha fechada tem realimentação. $\mathrm{O}$ sinal operante, que é a diferença entre o sinal de entrada e o sinal de saída da malha de controle, realimenta o controlador de modo a minimizar o erro, alcançando o valor desejado na saída.

Por meio de um diagrama de blocos, podese representar um sistema de controle automático, no qual todas as variáveis são interligadas por meio de blocos funcionais. São símbolos das operações matemáticas, que são aplicadas ao sinal de entrada, que produz o sinal de saída do bloco.

A função de transferência é utilizada para evidenciar as relações de entrada e saída de componentes, descritas por equações diferenciais lineares invariantes no tempo. As equações diferenciais são determinadas como a relação da Transformada de Laplace da saída como a função resposta, e a Transformada de Laplace da entrada como a função excitação, presumindo-se as condições iniciais iguais a zero.

O sistema de controle on/off, ou liga/desliga, consiste em ativar ou desativar o sinal de saída do controlador.

Os dispositivos elétricos dos sistemas de controle de duas posições permitem controlar, entre outras, a temperatura, a pressão, a umidade, a vazão e a velocidade. $\mathrm{O}$ intervalo diferencial é o intervalo no qual o sinal de erro atuante varia antes de ocorrer a comutação do sinal de ligado para desligado ou vice-versa.

\section{Metodologia POE}

A metodologia POE (Previsão, Observação e Explicação), segundo Sasaki (2017), é uma metodologia de aprendizagem ativa usada para o ensino de ciências exatas.

De acordo com Dos Santos (2015), a POE registra duas características principais. A primeira é viabilizar situações que estimulem o estudante a expor suas ideias, discutindo-as com seus colegas de classe, para depois apresentá-las de forma escrita. A segunda é que o centro da aula não é o professor, mas sim o aluno. O professor tem o papel de retratar os fenômenos, descrever o contexto do tema, incentivar a discussão de ideias, coordenar o diálogo com os alunos; porém, o aluno deve esclarecer e debater cada evento com suas próprias palavras.

A abordagem POE se desenvolveu na Universidade de Pittsburg e, no começo, foi nomeada DOE (Demostre, Observe e Explique). Foi proposta por Nedelsky (1961), White e Gunstone (1992) e citada por Barros (1994) (SCHWAHN; OIAGEN 2008).

Segundo Schwahn (2008) e White e Gunstone (2014), a metodologia é fundamentada em três etapas diversas. A primeira é a previsão, na qual se objetiva identificar as ideias individuais dos alunos. Nela os discentes são desafiados a debaterem o problema apresentado pelo docente, em equipes ou de forma individual, trocando experiências e apresentando hipóteses sobre o assunto com base nos conhecimentos adquiridos em sala de aula. O docente deve participar e estimular os alunos para que eles consigam responder adequadamente sobre o problema apresentado.

$\mathrm{Na}$ segunda etapa, denominada de observação, os discentes descrevem o conteúdo a ser analisado, realizam o experimento proposto pelo docente e registram suas observações, comparando-as com as ideias discutidas inicialmente. No final, todos debatem em grupos, conciliando os conflitos que surgiram entre a previsão e a observação e, assim, culminando o processo na etapa de explicação (WHITE; GUNSTONE, 2014).

Oliveira (2003) define a etapa da explicação como a mais importante, pois nessa ocasião aparece a resolução do problema inicial 
com base na contribuição das informações levantadas pelos grupos de alunos durante o primeiro e segundo momento. Nesse momento o professor assume o papel de mediador, conciliando os assuntos controversos para gerar resultados e, assim, explicar e solucionar o problema apresentado.

\section{METODOLOGIA}

Este estudo foi desenvolvido na Universidade do Vale do Taquari - Univates, com acadêmicos do curso de Engenharia Elétrica, na disciplina de Sinais e Sistemas. Os conteúdos trabalhados foram de função de transferência e sistemas de controle de primeira ordem.

Neste trabalho optou-se por utilizar análise descritiva dos resultados. Triviños (2008) afirma que as pesquisas em que a análise descritiva é utilizada conseguem relatam minunciosamente os fatos de uma determinada realidade, dos quais se obtém informações sobre o que se definiu como problema de pesquisa. Gil (2008), por sua vez, assegura que esse tipo de pesquisa contribui com novas visões de uma realidade. Ademais, essa técnica de análise tem como objetivo descrever os conjuntos de elementos que têm determinadas características semelhantes.

Segundo Alyrio (2008), a análise descritiva tem como objetivo ordenar dados, sem a finalidade de comprovar hipóteses exploratórias, gerando um espaço para um estudo justificado na experimentação. Barros e Lehfeld (2002) alegam que a análise dos resultados está diretamente relacionada com a criatividade do investigador, a sua compreensão sobre o conteúdo e o poder de argumentação.

Para tomada de informações recorreu-se a anotações, recursos fotográficos, gravações de áudios e filmagens, questionário de conhecimentos prévios, questionário sobre a percepção dos alunos e momento individual de aprendizagem.

As atividades foram divididas em seis momentos, totalizando três encontros, sendo um momento no primeiro encontro, dois momentos no segundo encontro e três momentos no terceiro encontro. No intervalo entre cada um dos encontros, o pesquisador se reuniu com seus orientadores para discutir os resultados, as dificuldades, pontos positivos e as melhorias para a próxima aula. Devido à pandemia (Covid-19), somente 50\% dos estudantes puderam participar do encontro presencial. Entretanto, toda aula foi transmitida ao vivo, por meio de vídeo chamada, o que possibilitou aos alunos de casa assistirem às aulas e interagirem com o professor e com a turma. A duração e os detalhes se encontram descritos a seguir:

a) $1^{\circ}$ momento: apresentação da proposta de pesquisa com os objetivos e as atividades planejadas; entrega do Termo de Consentimento Livre Esclarecido e assinado pelos alunos; entrega e preenchimento pela turma do Questionário de Conhecimento Prévio, o que permitiu ao professor planejar suas atividades seguintes, de forma mais adequada.

b) $2^{\circ}$ momento: realização de questionário oral com os alunos, com perguntas aleatórias para instigar os estudantes a participarem das aulas e responderem sobre o conteúdo trabalhado.

c) $3^{\circ}$ momento: realização de atividade experimental e abordagem teórica do conteúdo mediante a aplicação da metodologia POE. $\mathrm{Na}$ etapa da previsão, buscou-se, por meio de um questionário, conhecer as ideias iniciais dos estudantes sobre os conteúdos abordados. Na sequência, desenvolveuse os conteúdos de sistemas de controle de primeira ordem. A planta térmica utilizada na atividade experimental se concentrou em um sistema de aquecimento com base em uma lâmpada dicroica. A temperatura foi monitorada por intermédio de um sensor de temperatura modelo LM35, que apresenta uma saída de tensão linear relativo à temperatura. Para cada grau célsius que o sensor lê, ele varia $10 \mathrm{mV}$. $\mathrm{O}$ sensor possui três terminais: dois para alimentação, que pode ser de 4 a $20 \mathrm{~V}$, de acordo com a especificação técnica do fabricante; e o outro que é o sinal de 
saída analógica para conectar a entrada analógica de algum controlador. A planta térmica utilizada tem um transdutor de temperatura integrado em sua placa, com saída analógica de 0 a 10 V. Os estudantes construíram a curva e utilizaram a função de transferência c(t) para encontrar os valores de $\mathrm{t}$ para os tempos de T, 2T, 3T, 4T e 5T. A plataforma Arduino Uno foi utilizada para ler os valores da temperatura.

Os alunos tiveram que encontrar os valores de resistências adequados para o divisor de tensão. $\mathrm{O}$ professor apenas orientou os discentes para que pudessem construir sozinhos o conhecimento. Já para a leitura dos dados, o docente induziu os alunos a utilizarem a saída serial do Arduino. O código fonte utilizado para programação do controlador foi desenvolvido previamente pelo pesquisador, devido ao pouco tempo disponível para o fazê-lo em sala de aula.

A temperatura da estufa varia entre 29 a $73^{\circ} \mathrm{C}$. Os valores de temperatura da saída serial do Arduino foram exportados pelos alunos para uma planilha eletrônica, com leituras a uma taxa de leitura por segundo. Visando à correção de eventuais erros e variações, desenvolveu-se um algoritmo que realiza mil leituras e faz a média, gerando um valor confiável para a elaboração da tabela de valores e, com isso, obtendo-se valores mais precisos para o trabalho. Os dados na planilha foram normalizados, movendo o eixo $\mathrm{x}$ do gráfico até o ponto zero.

Com base no gráfico, analisou-se com os alunos os tempos de T, 2T, 3T, 4T e $5 \mathrm{~T}$ da função de transferência, envolvendo assim o sistema de primeira ordem. Findadas as atividades e discussões, realizou-se a explicação. Segundo Oliveira (2003), essa é a etapa mais importante da metodologia POE, na qual ocorre a resolução do problema com a contribuição dos grupos de alunos que participaram do primeiro e segundo momentos. Nesse momento os assuntos que geraram controvérsias foram conciliados com os alunos, culminado em resultados e na solução do problema inicial.

d) $4^{\circ}$ momento: realização de cálculos de sistema de controle de primeira ordem empregando LaPlace, que iniciou com um questionário para verificar $\mathrm{o}$ conhecimento dos alunos sobre os conteúdos trabalhados em sequência, com o intuito de aplicar a primeira etapa da metodologia POE, a previsão. Concluídas as discussões orais sobre essas questões, iniciou-se o conteúdo novo. $\mathrm{Na}$ sequência, realizou-se a dedução da função de transferência de um circuito RC genérico. Com a utilização da transformada de Laplace, empregando-se frações parciais, obteve-se a função de transferência. Aplicando-se a função degrau para a entrada, a Transformada de Laplace da função degrau é 1/S para esse sistema, o que permite chegar na função de transferência obtida pelo circuito $\mathrm{RC}$, que é similar à função da planta térmica analisada.

Obtida essa função, realizou-se questionamentos e reflexões com os alunos sobre o conteúdo desenvolvido até aquele momento e sobre os resultados encontrados.

e) $5^{\circ}$ momento: realização de atividades com simuladores computacionais, com a aplicação da segunda etapa do POE, a observação. Apresentou-se aos alunos o software de simulação Matlab, visando a estabelecer uma relação entre as atividades experimentais e os cálculos. Foram simulados sistemas lineares de respostas transitórias no tempo, em específico, a resposta da função degrau.

f) $6^{\circ}$ momento: realização do fechamento e conclusão de todas as atividades, mediante a aplicação da terceira etapa da metodologia POE, a explicação. Foram feitas reflexões com os alunos sobre o que foi abordado e o que eles acharam mais pertinente durante as aulas, com o registro dos pontos 
positivos e as melhorias para atividades futuras.

\section{RESULTADOS}

As atividades propostas nesse trabalho permitiram aos alunos trabalhar com conceitos de eletrônica analógica e digital, programação, sistemas de controle de primeira ordem, simulações computacionais, planilhas eletrônicas e cálculos matemáticos. A planta térmica e o uso do Matlab foram os pontos chaves dessa pesquisa, pois eles possibilitaram o ensino de sistemas de controle.

Com a aplicação da metodologia POE, criou-se uma sequência didática para o ensino de sistemas de controle de primeira ordem na disciplina de Sinais e Sistemas. Os questionários orais e escritos, bem como as percepções do professor, possibilitaram verificar os conhecimentos prévios dos estudantes. Já as atividades experimentais com a planta térmica em conjunto com as simulações computacionais no Matlab permitiram desenvolver essa metodologia de trabalho.

Nas atividades experimentais, a turma utilizou a planta térmica para gerar a curva de temperatura, simulando um sistema de primeira ordem. $\mathrm{O}$ equipamento, o qual os estudantes utilizaram para realizar as atividades, baseavase em um sistema de aquecimento por meio de uma lâmpada do tipo dicroica, em que a temperatura foi monitorada através de um sensor de temperatura modelo LM35. O aparato possuía um transdutor de temperatura integrado em sua placa, permitindo uma saída analógica de 0 a $10 \mathrm{~V}$. O objetivo principal da atividade era fazer com que os estudantes conseguissem construir uma curva de temperatura de um sistema de primeira ordem, semelhante à registrada por Ogata e Yang (2002) e apresentada na Figura 1.
Figura 1 - Curva exponencial de resposta de um sistema de primeira ordem

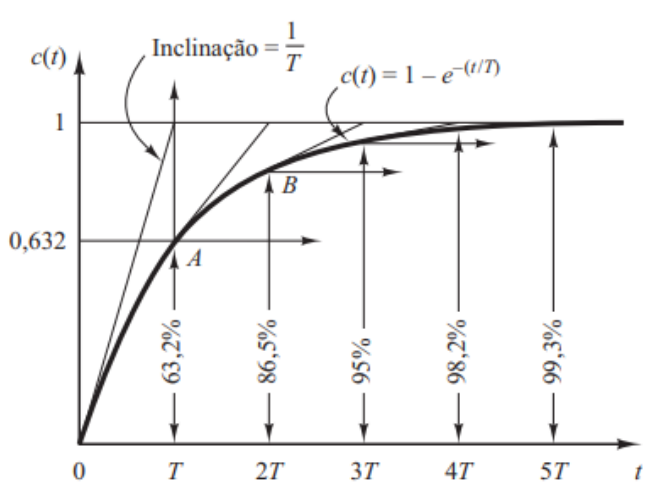

Fonte: Ogata e Yang (2002).

Após construírem a curva, os estudantes tiveram que utilizar a função de transferência $\mathrm{c}(\mathrm{t})$ para encontrar os valores de $\mathrm{t}$ para os tempos de T, 2T, 3T, 4T e 5T. Para leitura dos valores de temperatura foi utilizada a placa eletrônica do Arduino Uno. Entretanto, essa placa possui uma entrada analógica de 0 a $5 \mathrm{~V}$. Desse modo, para não sobrecarregar a entrada, os alunos tiveram que projetar um divisor de tensão para que, assim, a tensão de saída do sistema fosse minimizada.

Para desenvolver as atividades a turma se dividiu em três grupos: A, B e C. Porém, nenhum deles conseguiu fazer as leituras de forma correta na primeira tentativa. Os grupos A e B não conseguiam compilar o código de programação que foi disponibilizado para turma e se encontra no Apêndice $\mathrm{C}$ desse trabalho, pois a placa do Arduino que escolheram não estava operando. Após trocarem o Arduino, conseguiram ler os valores corretos de temperatura utilizando a saída serial. O grupo C conseguiu compilar o código, entretanto os valores lidos na saída serial estavam incorretos; nesse instante, verificaram que a estufa que eles escolheram estava com defeitos, provavelmente o conversor analógico digital do equipamento estava estragado. Dessa forma, depois de trocarem a planta térmica, conseguiram seguir com a atividade.

Depois de todos os grupos lerem os valores e exportarem para a planilha eletrônica, fazendo a normalização dos dados, os grupos A, B e C conseguiram obter, respectivamente, os gráficos abaixo, apresentados nas Figuras 2, 3 e 4. 
Figura 2 - Gráfico de temperatura do grupo A

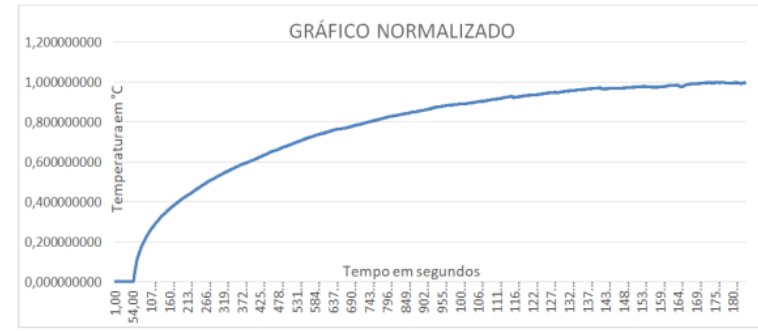

Fonte: elaborada pelos autores, 2020.

Figura 3 - Gráfico de temperatura do grupo B Gráfico Normalizado: Temperatura (ํ) X Tempo (s)

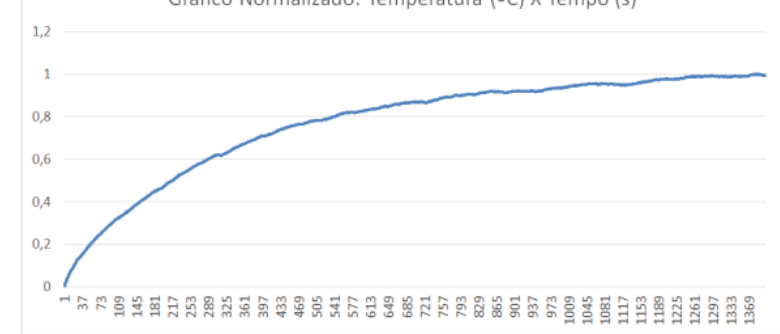

Fonte: elaborada pelos autores, 2020.

Figura 4 - Gráfico de temperatura do grupo C

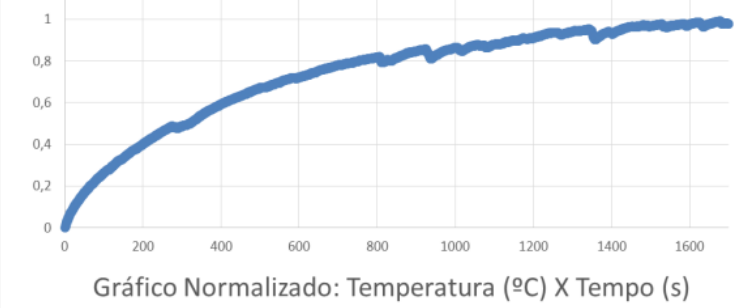

Fonte: elaborada pelos autores, 2020.

Conforme pode ser observado, todos os alunos conseguiram obter gráficos semelhantes ao da Figura 1, do livro do Ogata e Yang (2002). Em sequência, os acadêmicos utilizaram a função de transferência $\mathrm{c}(\mathrm{t})$ para encontrar os valores de t para os tempos quando $\mathrm{a}=\mathrm{T}, \mathrm{a}=2 \mathrm{~T}, \mathrm{a}=3 \mathrm{~T}, \mathrm{a}=4 \mathrm{~T}$ e $\mathrm{a}=5 \mathrm{~T}$.

Tanto os valores de tempo quanto os de temperatura encontrados pelos grupos equivalem a primeira constante de tempo que vai de 0 a $63,2 \%$ da resposta final, a segunda constante de tempo que representa $86,5 \%$ do valor final, a terceira, quarta e quinta constantes de tempo que correspondem, respectivamente, a $95 \%, 98,2 \%$ e $99,3 \%$ da resposta final (OGATA; YANG, 2002).

Após realizarem as atividades os alunos comentaram o seguinte: "[...] professor, através da planta térmica, pude compreender o comportamento de um sistema de primeira ordem, pois, no início, quando o senhor apresentou esse sistema para nós, ele parecia algo muito abstrato". Outro deles comentou: "[...] como eu não trabalho na área, para mim fica muito difícil conseguir visualizar como um sistema de primeira ordem funciona, através dessa atividade que fizemos pude ter mais noção". Outros alunos também mencionaram: "[...] no início eu achei que iria ser muito dificil conseguir chegar nessa curva e durante a atividade tive muitas dificuldades, porém no final tudo deu certo, estou muito feliz em conseguir visualizar como esse sistema funciona".

De acordo com os comentários dos estudantes e com a percepção que se teve durante todo o processo, pode-se dizer que no início os alunos acharam tudo muito confuso. Eles tiveram várias dificuldades, como o Arduino que não funcionava; o divisor de tensão o qual não conseguiram construir corretamente na primeira tentativa; a estufa que não transmitia os dados corretamente; e erros com a planilha em função dos alunos inserirem as fórmulas erradas. Porém, com a capacidade investigativa que a turma teve em conjunto com todo o apoio do professor, todos os estudantes conseguiram desenvolver as atividades propostas.

O momento individual de aprendizagem, em conjunto com questionário de percepção e com o olhar crítico e atento do docente, permitiu conhecer as percepções dos discentes frente a todas as atividades realizadas. $\mathrm{O}$ Quadro 1 integra as principais ações desenvolvidas nesta pesquisa, acompanhadas de seus resultados. 
Quadro 1 - Quadro integrador das principais ações realizadas e seus resultados

\begin{tabular}{|c|c|}
\hline Ação & Resultado \\
\hline $\begin{array}{c}\text { Questionários de } \\
\text { conhecimentos prévios. }\end{array}$ & $\begin{array}{c}\text { Observou-se que os alunos apresentaram bons } \\
\text { conhecimentos prévios na teoria de sistemas de } \\
\text { controle de primeira ordem, entretanto nos cálculos } \\
\text { matemáticos tinham dificuldades. }\end{array}$ \\
\hline $\begin{array}{c}\text { Atividade experimental com } \\
\text { a planta térmica. }\end{array}$ & $\begin{array}{c}\text { Os discentes desenvolveram conceitos de eletrônica } \\
\text { analógica e digital, programação, sistemas de } \\
\text { controle de primeira ordem, planilhas eletrônicas e } \\
\text { cálculos matemáticos. Provavelmente, através da } \\
\text { atividade experimental, compreenderam melhor o } \\
\text { funcionamento de um sistema de controle de } \\
\text { primeira ordem. }\end{array}$ \\
\hline $\begin{array}{c}\text { Atividade de simulação no } \\
\text { software Matlab. }\end{array}$ & $\begin{array}{c}\text { Desenvolveu-se com os alunos conceitos de } \\
\text { programação, simulação computacional e } \\
\text { aprofundou-se com eles o entendimento do } \\
\text { funcionamento de um sistema de controle de } \\
\text { primeira ordem e visualizou-se sistemas de } \\
\text { diferentes ordens. }\end{array}$ \\
\hline $\begin{array}{c}\text { Atividade de dedução da } \\
\text { função de transferência, via } \\
\text { cálculos matemáticos, de um } \\
\text { circuito RC. }\end{array}$ & $\begin{array}{c}\text { Aperfeiçoou-se com os alunos os conceitos de } \\
\text { cálculos matemáticos, principalmente aqueles, } \\
\text { onde eles tinham mais dificuldades no início da } \\
\text { intervenção pedagógica. }\end{array}$ \\
\hline Avaliação individual. & $\begin{array}{c}\text { Verificou-se que os alunos entenderam os cálculos } \\
\text { matemáticos de um sistema de controle de } \\
\text { primeira ordem. }\end{array}$ \\
\hline percepção. & $\begin{array}{c}\text { Os alunos se manifestaram de forma positiva com } \\
\text { o desenvolvimento das atividades. }\end{array}$ \\
\hline & \\
\hline
\end{tabular}

Fonte: elaborado pelos autores, 2020.

Analisando os resultados obtidos por meio das ações realizadas nesse trabalho, observa-se que elas vão ao encontro dos objetivos desta pesquisa.

Ao analisar o questionário de conhecimentos prévios, em que a turma teve que responder três perguntas, a respeito de um sistema de primeira ordem, de controle de processos, pôde-se verificar que os alunos apresentavam conhecimentos satisfatórios sobre a teoria de controle. Entretanto, na parte de cálculos, quando foi solicitado para os estudantes desenvolverem uma equação diferencial, menos de $30 \%$ da turma conseguiu acertar a questão. Diante disso, evidenciou-se a necessidade de dar mais ênfase na parte de cálculos. Assim, no terceiro encontro foi realizado, detalhadamente, toda a dedução numérica de um sistema de controle de primeira ordem de um circuito $\mathrm{RC}$, com a revisão das frações parciais, comparando-se a resposta do sistema com a já conhecida da planta térmica, desenvolvida pelos alunos em momentos anteriores.

No primeiro momento, os alunos observaram a dedução solução de um circuito RC genérico. Com o objetivo de chegar nessa solução, recorreu-se à Transformada de Laplace, aplicando-se as frações parciais. A equação que determina o circuito RC é análoga ao da planta térmica na qual os alunos trabalharam anteriormente; desse modo, apenas visualizaram matematicamente a dedução para chegar a tal equação, oportunizando uma nova maneira de compreender esse mesmo conteúdo.

Essa modelagem matemática permitiu deduzir a solução de um circuito Resistivo Capacitivo genérico no domínio da frequência, obtendo-se a resposta no domínio do tempo. Para resolução do sistema, aplicando-se a 
função degrau para a entrada, a Transformada de Laplace da função degrau é $1 / \mathrm{S}$. Dessa forma, as equações a seguir apresentam os cálculos passo a passo do circuito RC.

$$
\begin{array}{cc}
V s-i R-\frac{q}{C}=0 & \text { Eq. (1) } \\
X(t)=V s . u(t) ; Y(t)=q & \text { Eq. (2) } \\
X(t)=R y^{\prime}+\frac{Y}{C} & \text { Eq. (3) } \\
R s Y(s)^{\prime}+\frac{Y(s)}{C}=X(s) & \text { Eq. (4) } \\
Y(s) \cdot\left(R(s)+\frac{1}{C}\right)=X(s) & \text { Eq. (5) } \\
R s Y(s)^{\prime}+\frac{Y(s)}{C}=X(s) & \text { Eq. (6) } \\
X(s)=\frac{1}{s} & \text { Eq. (7) } \\
Y(s)=\frac{X(s)}{R s+\frac{1}{C}} & \text { Eq. (8) } \\
Q(t)=\frac{1}{\frac{R}{R} \cdot\left(R s+\frac{1}{C}\right)} \cdot \frac{V s}{s}=\frac{1}{R} \cdot\left(\frac{1}{s+\frac{1}{R C}}\right) \cdot \frac{V s}{s}= \\
\frac{V s}{R} \cdot\left(\frac{a 1}{\left.s+\frac{1}{R C}+\frac{a 2}{s}\right)}\right. & \text { Eq. (9) }
\end{array}
$$

A partir da técnica de frações parciais ${ }^{6}$ conseguiu-se continuar a resolução, obtendo-se as Equações seguintes.

$$
\begin{array}{r}
Q(t)=\frac{V s}{R} \cdot\left(\frac{-R C}{s+\frac{1}{R C}}+R C \cdot \frac{1}{s}\right)= \\
\frac{V s}{R} \cdot\left(-R C \cdot e^{\frac{-t}{R C}}+R C \cdot 1\right) \\
Q(t)=C \cdot V s\left(1-e^{\frac{-t}{R C}}\right) \\
i(t)=\frac{d Q}{d t}=C \cdot \frac{V s}{R C} \cdot\left(1-e^{\frac{-t}{R C}}\right)
\end{array}
$$

Para finalizar, foram multiplicados os termos por R, obtendo-se a Equação do circuito RC no domínio do tempo, que é apresentada pela Equação 13:

$$
V(t)=V s \cdot\left(1-e^{\frac{-t}{R C}}\right)
$$

Essa solução do sistema, obtida através do circuito Resistivo Capacitivo, é semelhante à solução do sistema da planta térmica, a qual os alunos obtiveram na atividade experimental realizada.

Ao analisar o comportamento de ambas as curvas, a turma chegou à conclusão de que a curva de carga do circuito RC representa uma função exponencial, da mesma forma que a da planta térmica. Entretanto, a constante de tempo do circuito RC é $R$. $C$, enquanto da planta térmica é $T$. Observando as curvas a turma constatou que quanto menor for a constante de tempo, mais rápida é a resposta do sistema. Além disso, puderam confirmar que a resposta de inclinação da linha tangente da curva exponencial é $1 / T$, quando t é igual a 0 , de acordo com o livro de Ogata e Yang (2002).

\section{CONCLUSÕES}

A utilização de atividades experimentais em conjunto com a aplicação da metodologia da Previsão, Observação e Explicação contribuiu para aumentar a predisposição dos estudantes para aprender. A situação foi verificada em todas as etapas da intervenção pedagógica e confirmada por meio do questionário sobre a percepção dos alunos, no qual a maioria da turma registrou que as atividades realizadas contribuíram para facilitar o entendimento, com a aplicação na prática dos conteúdos de sinais e sistemas.

O uso de uma planta térmica, comparandoa com um circuito Resistivo Capacitivo, motivou os alunos a saírem da zona de conforto e a buscarem o conhecimento.

Os alunos foram estimulados a participarem de todos os momentos propostos nessa sequência didática.

${ }^{6}$ Cf. OGATA, K.; YANG, Y., 2002, p. 147. 
Por meio da pesquisa, verificou-se como utilizar as atividades experimentais e computacionais, por meio da metodologia POE, nos conteúdos de sistemas de controle de primeira ordem, na disciplina de Sinais e Sistemas, do curso de Engenharia Elétrica. Identificou-se que a utilização de sistemas reais, como a planta térmica, em conjunto com uma metodologia de aprendizagem ativa, proporcionou o aumento do interesse dos estudantes em aprender os conteúdos.

A parceria que se estabeleceu entre o docente e os estudantes contribuiu para que estes se tornassem sujeitos ativos durante as atividades realizadas, fazendo com que suas participações tivessem grande importância para o resultado desejado. Desde os questionários introdutórios das aulas, em que a turma teve que presumir o comportamento de um sistema de primeira ordem e no qual foi empregado a primeira etapa da POE (a Previsão), até o levantamento de um gráfico da planta térmica, em que os estudantes tiveram que desenvolver um meio de ler o sinal de tensão do sistema e transferi-lo para uma planilha eletrônica, processo no qual se aplicou a segunda etapa do POE (a Observação) e posteriormente a terceira etapa (a Explicação), a turma foi instigada a participar dinamicamente de todas as atividades propostas.

Os dados analisados a partir desse trabalho mostraram que: a) os alunos possuíam bons conhecimentos prévios sobre sinais e sistemas, entretanto faltava-lhes a integração de conhecimento entre a teoria de controle e os cálculos matemáticos; b) a construção das atividades experimentais, voltada tanto para teoria quanto para a prática da engenharia, possibilitou a turma desenvolver habilidades e competências para realizar, de forma autônoma, a solução de problemas de controle de sistemas de primeira ordem; e c) a metodologia POE se fez presente em todos os momentos, o que propiciou a aprendizagem e estabeleceu a parceria entre professor e alunos na busca do conhecimento. $\mathrm{O}$ uso das atividades experimentais aplicadas aos problemas reais permitiu o ensino de Sinais e Sistemas.

Como sugestão para futuros trabalhos, instiga-se que novos pesquisadores desenvolvam com suas turmas outros tipos de sistemas de controle, como pêndulo invertido, servossistemas de velocidade e atenuação de ruído, empregando-se sistemas de segunda ordem.

\section{CONCLUSÕES}

De acordo com os indicadores da qualidade formulados pelo MEC, apenas 5\% dos cursos de Engenharia Florestal no Brasil apresentam Conceito Preliminar do Curso 5-que é demonstrativo de excelência e referência no país. Tal situação evidencia que, apesar do conceito satisfatório alcançado, muitos cursos ainda precisam melhorar seus níveis de qualidade exigidos pelo sistema de avaliação da qualidade determinado pelo Governo Federal. Esse fator pode ser interpretado como uma oportunidade de melhoria e deve ser inserido como meta de longo prazo no planejamento estratégico do curso.

\section{REFERÊNCIAS}

ALEXANDER, D.; HAYSOM, J.; BOWEN, M. Predict, observe, explain: activities enhancing scientific understanding. NSTA Press, 2010.

ALYRIO, R. D. Metodologia científica. PPGEN: UFRRJ, 2008.

BANZI, M.; SHILOH, M. Primeiros passos com o Arduino. São Paulo: Novatec, 2011.

BARROS, A. J. P. de; LEHFELD, N. A. S. Projeto de pesquisa: propostas metodológicas. Petrópolis: Vozes, 2002.

DEMO, P. Educar pela pesquisa. São Paulo: Autores Associados, 1998.

DOS SANTOS, L. G. F; NEIDE, I. G.; REHFELDT, M. J. H. O ensino de automação: ambiente escolar automatizável por meio do desenvolvimento de um painel eletroeletrônico centralizado. Revista Thema, v. 14, n. 3, p. 7787, 2017.

DOS SANTOS, R. J.; SASAKI, D. G. G. Uma metodologia de aprendizagem ativa para o ensino de mecânica em educação de jovens e 
adultos. Caderno Brasileiro de Ensino de Física, v. 37, n. 3, 2015.

OGATA, K.; YANG, Y. Modern control engineering. London: Prentice Hal, 2002.

OLIVEIRA, P. R. S. A construção social do conhecimento no ensino e aprendizagem de Química. Anais... IV Encontro Nacional de Pesquisa em Educação em Ciências, 4, Bauru: ABRAPEC, 2003.

NEIDE, I. G.; QUARTIERI, M. T. Recursos tecnológicos nos processos de ensino e de aprendizagem da Matemática e da Física. In: QUARTIERI, M. T.; DULlIUS, M. M. Aproximando a Matemática e a Física por meio de recursos tecnológicos: Ensino Médio. Lajeado: Ed. da Univates, 2016.

SASAKI, D. G. G.; DE JESUS, V. L. B. Avaliação de uma metodologia de aprendizagem ativa em óptica geométrica através da investigação das reações dos alunos. Revista Brasileira de Ensino de Física, v. 39, n. 2, 2017.
SCHWAHN, M. C. A.; OIAGEN, E. R. O uso do laboratório de ensino de Química como ferramenta: investigando as concepções de licenciandos em Química sobre o Predizer, Observar, Explicar (POE). Acta Scientiae, v. 10, n. 2, p. 151-169, 2008.

TEODORO, V. D. K.; VEIT, E. A. Modelagem no ensino/aprendizagem de física e os novos parâmetros curriculares nacionais para o ensino médio. Revista Brasileira do Ensino de Física, São Paulo, v. 24, n. 2, p. 86-96, Jun. 2002.

TRIVIÑOS, A. N. S. Introdução à pesquisa em ciências sociais: a pesquisa qualitativa em educação. São Paulo: Atlas, 2008.

WHITE, R.; GUNSTONE, R. Probing understanding. London: Routledge, 2014.

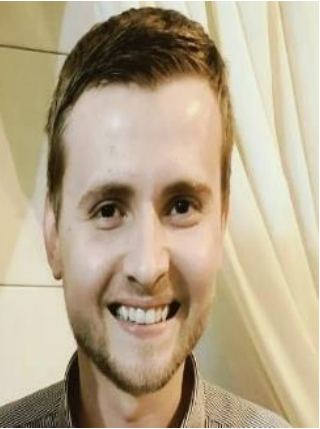

\section{DADOS BIOGRÁFICOS DOS AUTORES}

Mateus Vorpagel - Engenheiro Eletricista (CREA RS236435) formado em 2018 pela Universidade do Vale do Taquari - Univates. Graduado em Matemática (Licenciatura) pela Universidade do Norte do Paraná - Unopar. Cursando PósGraduação em Metodologias Ativas e TDICs na Educação na Universidade do Norte do Paraná - Unopar. Cursando Mestrado em Ensino de Ciências Exatas na Universidade do Vale do Taquari - Univates. Trabalha como professor de Matemática nos anos finais do Ensino Fundamental na rede municipal de educação. Atua, de forma autônoma, desenvolvendo projetos de eletricidade industrial nas normas técnicas. Ministra cursos de formação continuada na Universidade do Vale do Taquari - Univates, como por exemplo Curso de PCI utilizando software Cad.

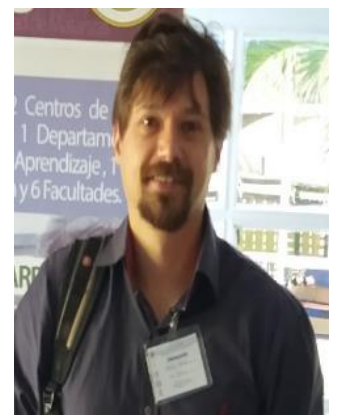

Ítalo Gabriel Neide - Possui Bacharelado (2004) e Mestrado (2007) em Física e Doutorado em Ciências (ênfase Física Teórica) (2011) pela Universidade Federal do Rio Grande do Sul. Pós-Doutor pela Universität Duisburg-Essen. Desenvolveu colaborações na PUC-Chile e University of New Mexico. Atualmente é professor da Universidade do Vale do Taquari - Univates, atuando nos cursos de graduação e coordenador do Programa de Pós Graduação em Ensino de Ciências Exatas (nível mestrado e doutorado). Tem experiência na área de Física da Matéria Condensada, Modelos Teóricos Computacionais, Ensino de Física e Modelagem Matemática, atuando principalmente nos seguintes temas: uso de tecnologias no ensino de Física, integração de atividades práticas e computacionais para o ensino de Física, modelagem científica e Design Based Research. 


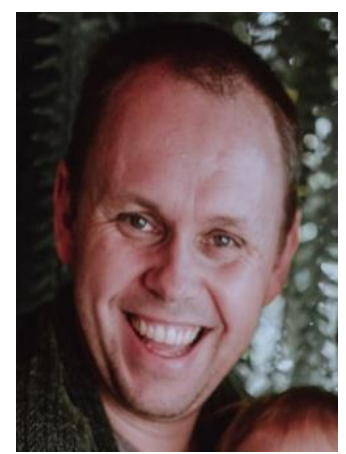

Juliano Schirmbeck - Possui doutorado em Sensoriamento Remoto com aplicação em Agrometeorologia pela Universidade Federal do Rio Grande do Sul (UFRGS 2017). Obteve o título de mestre em Geologia com ênfase em Gerenciamento Ambiental pela Universidade do Vale do Rio dos Sinos (UNISINOS - 2005). Graduado em Engenharia Elétrica pela Universidade do Vale do Rio dos Sinos (UNISINOS - 2002). Professor dos cursos de Engenharia Ambiental e Engenharia Elétrica na Universidade do Vale do Taquari (UNIVATES). Especialista em programação para processamento digital de imagens com conhecimento nas linguagens IDL no Envi, Java Script-Python no Google Earth Engine, Python e R Studio. Tem experiência em ensino e pesquisa em engenharia, sensoriamento remoto e agrometeorologia, atuando principalmente nos seguintes temas: balanço de energia e estimativa de: evapotranspiração, temperatura de superfície, deficiência hídrica com imagens de satélite, programação para análise de séries temporais de imagens e elementos meteorológicos. Atua como professor em disciplinas de Modelagem e Simulação ambiental, Geoprocessamento aplicado a recursos hídricos, Climatologia e modelagem e Controle de processos. 